\title{
CAMBIO Y GENERACIÓN de capacidades competitivas Un estudio en las empresas medianas y grandes de confecciones del eje cafetero
}

\section{RESUMEN}

Este artículo pretende constatar las relaciones entre el cambio organizacional y las capacidades organizativas críticas para la competitividad, relaciones que la literatura especializada define desde un enfoque teórico. Se seleccionaron seis capacidades críticas: flexibilidad, gestión del capital humano, liderazgo, aprendizaje organizacional, relaciones con el cliente y claridad estratégica. En este trabajo estas relaciones se analizan desde la teoría de recursos y capacidades.

La muestra se cotejo aplicando el cuestionario propuesto a 35 directivos de siete empresas medianas y grandes del sector confecciones del Eje Cafetero; posteriormente se realizó una entrevista con cinco expertos del sector para analizar los hallazgos. Se encontró evidencia empírica para afirmar que el cambio organizacional incide en el desarrollo de las capacidades críticas competitivas.

\section{palabras clave}

Cambio, capacidades organizativas, competitividad, teoría de recursos y capacidades, sector confecciones.
Por:

Paula Andrea Cruz Mejía

Gregorio Calderón Hernández ${ }^{1}$

$\mathbf{E}$ cambio se ha convertido en una variable estratégica en el desarrollo de las organizaciones (Motta, 2001), por cuanto se espera que las empresas sometidas a procesos de transformaciones aumenten su nivel competitivo al incorporar nuevas tecnologías y desarrollar capacidades organizativas críticas (Blumental y Haspeslagh, 1994, p. 101).

Las capacidades organizativas críticas son aquellas que facilitan la construcción de una ventaja competitiva y se constituyen en una habilidad para desplegar y aprovechar los recursos en busca de fines empresariales y el logro de un desempeño superior (Amit y Schoemaker, 1993, p. 14, 33-46; Lado y Wilson, 1994, p. 669-727). La revisión de la literatura especializada permite pensar que al menos seis capacidades están asociadas con el cambio: flexibilidad, gestión del capital humano, liderazgo, aprendizaje organizacional, relaciones con el cliente y claridad estratégica.

La inquietud teórica acerca de la relación entre cambio y capacidades críticas, más las características propias de un sector de la economía regional-el sector

\footnotetext{
1 Paula Andrea Cruz Mejía. Ingeniera Industrial, especialista en Desarrollo Gerencial, Magíster en Administración. Gregorio Calderón Hernández. Profesor Asociado Universidad Nacional de Colombia, Sede de Manizales. Director de grupo de investigación en cultura organizacional y gestión humana reconocido por Colciencias. Magister en Administración, candidato a doctor en Administración y Dirección de Empresas por la Universidad Pablo de Olavide, Sevilla.
} 
de las confecciones, que hace un aporte significativo al PIB nacional y que por sus condiciones ha estado bajo una fuerte presión de cambio en los últimos años-, motivaron la investigación base del presente artículo a partir de la pregunta: ¿los procesos de adaptación al cambio que han debido asumir las empresas de confecciones en los últimos años han desarrollado en ellas capacidades organizacionales que les permitan construir ventajas competitivas sostenidas?

Esta investigación hace parte del macroproyecto «Gestión Humana y Estrategia Empresarial en Colombia», que desde 2002 adelanta el grupo de investigación Cultura Organizacional y Gestión Humana de la Universidad Nacional de Colombia Sede Manizales, reconocido y categorizado por Colciencias; esta investigación se llevó a cabo entre enero de 2005 y mayo de 2006.

Metodológicamente se optó por un diseño cuantitativo de tipo explicativo en el cual la variable independiente (cambio) puede explicar la existencia de algunas capacidades organizativas (variables dependientes), con base en la teoría de recursos y capacidades (Barney, 1991, p 99-120; Teece, et.al., 1997, p. 509-533).

La técnica empleada fue la encuesta, aplicada a 35 directivos de siete empresas medianas y grandes del sector confecciones del Eje Cafetero. Como mecanismo para garantizar validez y confiabilidad se realizó un análisis de las preguntas a partir de marco teórico y se realizó un pretest con cinco profesionales del sector. El cuestionario fue complementado con una entrevista a cinco expertos del sector, con el fin de contrastar los resultados arrojados por la encuesta. La prueba de hipótesis se hizo a través del análisis de varianza Anova.

Los resultados muestran que existe evidencia empírica para pensar que capacidades organizativas como: flexibilidad, gestión del capital humano, liderazgo, aprendizaje organizacional, relaciones con el cliente y claridad estratégica están relacionadas con el cambio.

\section{A Bstract}

From a quantitative perspective, applying the variance analysis it is intended to verify the relationships between the organizational change and the organizational capabilities that are critic to competitiveness; relationships that were theoretically found in the specialized literature. Six capabilities were selected: flexibility, management of the human capital, leadership, organizational learning, relationships with the client and strategic clarity. The resources and capabilities theory was assumed to understand the named relationships.

The comparison was made applying a questionnaire to 35 managers of seven medium and big enterprises of the tailoring sector of the Eje Cafetero region; later on an interview was conducted with five experts from the sector to analyze the findings. Empirical evidence was found in order to affirm that the organizational change does influence the development of the competitive critic capabilities.

\section{KEY WORDS}

Change, organizational capabilities, competitiveness, theory of resources and capabilities, tailoring sector. 


\section{METODOLOGÍA DE LA INVESTIGACIÓN}

\subsection{El problema de investigación}

El proceso de globalización sumado a los desarrollos tecnológicos generados especialmente por la inserción de tecnologías de la información a los procesos productivos y la apertura de mercados han generado un fuerte impacto en todos los sectores económicos produciendo nuevos desafíos gerenciales. «La globalización ha estimulado la competitividad desde tres ángulos: la búsqueda permanente del aumento de valor para el cliente, la reducción de los costos unitarios de producción y la extracción de competitividad de la sociedad» (Mendoza, 2000, p. 68-82).

El sector de las confecciones, estrechamente ligado con la moda, es uno de los más afectados tanto por el incremento y cualificación de su tecnología productiva, como por la llegada de competidores globalizados que han cambiado el escenario de los negocios, replanteando la estructura de precios y de calidad y requiriendo un mayor valor agregado en los productos $y$ también en el soporte proveedor-cliente.

En estas circunstancias las empresas del sector se han visto precisadas a asumir los cambios que el entorno exige o a desaparecer del mercado. Sin embargo, no basta con ser reactivos a los cambios en aspectos de productividad, eficiencia, mayor tecnología o innovación en diseños si paralelo a ello la empresa no genera nuevas capacidades de la organización que le sirvan de soporte para una ventaja competitiva sostenida en el mediano y largo plazo (Ventura, 1996).

Algunas de estas capacidades de la organización están relacionadas con las personas, específicamente con sus competencias y su posibilidad de aplicación de nuevos conocimientos (Boxall, 1996, p. 5975). Este factor está ligado al tipo de liderazgo impulsado en la organización, así como a la capacidad de flexibilidad para responder oportunamente a los cambios (Grewal y Tansuhaj, 2001), a la construcción de mejores relaciones con los clientes y a la claridad estratégica (Hamel y Prahalad, 1995).

Las mencionadas circunstancias se constituyeron en la base de la pregunta de investigación que dio origen al presente trabajo: ¿Los procesos de adaptación al cambio que han debido asumir las empresas de confecciones en los últimos años han desarrollado en ellas capacidades organizacionales que les permitan construir ventajas competitivas sostenidas?

La respuesta a esta inquietud plantea dos finalidades: desde el punto de vista teórico, interesa conocer si existe relación entre el cambio y algunas capacidades organizacionales; desde lo empírico, se pretende constatar cómo se da la relación cambiocapacidades en el sector de confecciones, específicamente en las empresas medianas y grandes, del Eje Cafetero colombiano.

En concordancia con lo anterior la hipótesis se formuló de la siguiente manera:

Ho: La adaptación al cambio que han vivido las empresas de confecciones del Eje Cafetero en los últimos seis años ha generado el desarrollo de capacidades tales como la flexibilidad, el desarrollo del capital humano, el liderazgo, el aprendizaje organizacional, las relaciones con el cliente y la claridad estratégica. 


\subsection{Diseño general de investigación}

Se asume como perspectiva metodológica general la investigación causal (Namakforoosh, 2002) con la que se pretende constatar que una variable independiente, el ambiente de cambio, afecta a la variable dependiente capacidades organizacionales.

Se definió como unidad de análisis las empresas medianas y grandes de confecciones del Eje Cafetero (que en total son 15) ${ }^{1}$ y como unidad de observación los gerentes, jefes de línea y directores de recursos humanos de estas empresas ( 60 en total) dada su relación directa con el tema de investigación y su rol protagónico en las unidades productivas.

Se adoptó un enfoque cuantitativo. Se diseñó un cuestionario integrado por dos bloques, el primero de 10 preguntas destinadas a establecer las características sobre el ambiente de cambio vivido por la empresa en los últimos seis años, en relación con tecnologías, nuevos productos, fusiones, cambios culturales, cambios en gestión, en estructura y en mercados. Se emplearon preguntas cerradas con escala aditiva de Likert con alternativas desde 1 (nunca se han presentado) hasta 5 (se han presentado en gran medida).

El segundo bloque, integrado por 18 ítems, estaba encaminado a identificar posibles capacidades que hubieren surgido en el mismo lapso. Con base en el marco teórico se escogieron seis posibles capacidades y se formularon tres ítems por cada una para que los encuestados calificaran el desarrollo de la respectiva capacidad desde 1 (baja) hasta 5 (alta).

El cuestionario fue sometido a un pretest con cinco jefes de área del sector con el fin de analizar la confiabilidad del instrumento y de obtener una varianza que permitiera el cálculo del tamaño de la muestra. Con un nivel de confianza del $95 \%$ y un error muestreal del $10 \%$ se estableció una muestra de 35 cargos a los cuales se les aplicó el cuestionario.

Además se realizó una triangulación (Valles, 1999), para complementar el enfoque cualitativo, con cinco entrevistas a sendos directivos empresariales del sector que no hicieron parte de la muestra con un cuestionamiento único: su percepción sobre la incidencia del cambio en la generación de cada una de las capacidades escogidas.

Para la verificación de hipótesis se utilizó un análisis de varianza (Anova) con la prueba de Fisher. Se escogió este parámetro por ser más comprehensivo que la razón t (student) por cuanto el Anova considera la varianza total de todos los sujetos en una muestra incluyendo la varianza entre los grupos y la varianza dentro de los grupos.

\footnotetext{
1 Durante el período de investigación cuatro de estas empresas se vieron abocadas a fuertes crisis, en algunos casos de cierre o amenaza de cierre.
} 


\section{CONTEXTO DE LA INVESTIGACIÓN}

En Colombia la industria de confecciones genera alrededor de 600.000 empleos directos e indirectos y exportó US $\$ 788$ millones en el año 2000 con destino a Estados Unidos (49\%), Latinoamérica (40\%) y Europa (11\%) (Vivianco, et.al., 2002).

En general, la industria nacional del sector no es competitiva frente a la producción china y ante la Iniciativa de los miembros de la Cuenca del Caribe (CBI) que son sus principales competidores en USA, pues los primeros tienen grandes ventajas en el costo de la mano de obra y los segundos son maquiladores con poco valor agregado y, por lo tanto, con costos reducidos por mano de obra no calificada. Estos últimos cuentan además con ventajas arancelarias de las cuales carece la industria colombiana. Sin embargo, la confección colombiana se ubica en un nicho de productos más elaborados: blue jeans, vestidos de dama, ropa infantil y ropa interior, con mayor valor agregado.

Por las condiciones de precio de las materias primas, de maquinaria disponible, de cargas salariales y de impuestos y transportes, el sector de la confección en Colombia sólo es rentable si trabaja con más de un $60 \%$ de eficiencia; para alcanzar este nivel, según los empresarios, un empleado sin la formación adecuada puede requerir entre tres o cuatro años en un puesto de trabajo ${ }^{1}$.
La industria de la confección en el Eje Cafetero concentra unas 1400 empresas la mayoría de ellas micros y pequeñas empresas, pues tan sólo 15 son consideradas medianas o grandes (con 50 o más trabajadores vinculados); la industria de la región representa cerca del $20 \%$ de las empresas y del empleo del sector en todo el país. Desde 1997 la industria de mayor tamaño del Eje Cafetero presenta incrementos anuales en las exportaciones, a la par con disminuciones progresivas de las utilidades de las empresas, lo cual ha producido una reducción progresiva de las inversiones.

Los empresarios señalan como principales causas de este fenómeno las tendencias de la industria, que da preferencia a pedidos de pequeño volumen (que disminuyen las ventajas de la producción en escala), con cambios frecuentes en las especificaciones (que demanda transformaciones internas permanentes) y cortos períodos para la entrega de pedidos (exigencia de menores ciclos de producción y flexibilidad en las entregas).

A esto se agregan problemas de alta rotación de la mano de obra calificada entre las empresas, lo cual obliga al mediano y gran empresario a estar formando permanentemente personas, a una elevada rigidez y resistencia al cambio entre las personas calificadas para la polivalencia y a costos asociados con las condiciones de trabajo.

\footnotetext{
1 Cálculos efectuados con base en los conceptos de los expertos.
} 


\section{MARCO TEÓRICO}

\subsection{Cambio y organizaciones}

En la presente investigación se asume el cambio organizacional como una alteración de las características socio-estructurales de la organización que trae como consecuencia modificaciones en el comportamiento de los integrantes, el portafolio de servicios y productos y la forma de gestión. Si bien muchas veces la fuente motivadora del cambio es externa, es importante aclarar que para efectos de esta investigación solamente se considera que se ha dado un cambio organizacional cuando se genera una transformación interna con efectos sobre los resultados y la dinámica de la organización.

Las organizaciones se reconocen como sistemas abiertos que mantienen permanentes transacciones con su ambiente, el cual influye en su supervivencia y progreso (Motta, 2001): aquellas que sobreviven se vuelven fuertes en cuanto encuentran ventajas diferenciadoras que las hacen más competitivas (Hanna y Freeman, 1977, p. 929-964); es decir, aquellas que no solo mejoran su velocidad reactiva sino que aprovechan las nuevas oportunidades que los cambios continuos del entorno les ofrecen «...sistemáticamente buscan indicios tempranos de nuevas capacidades que pudieran permitirles responder mejor a las necesidades que se hayan identificado" (Haeckel, 2002, p. 77-95).

En este proceso de adaptación a los cambios generados por fuerzas externas se espera que la empresa aumente su nivel competitivo, al incorporar avances tecnológicos, aumentar la eficiencia y crear capacidades tecnológicas que a su vez la hagan más flexible para futuras transformaciones (Child y Kieser, 1981).
En la teoría ecológica de las organizaciones (Hanna y Freeman, 1977; Kelvey y Aldrich, 1983, p. 101-128) se encuentran algunas características del cambio empresarial que son de interés en la presente investigación; en primer lugar el denominado principio de la variación con propósito, esto es, que las presiones del medio ambiente fomentan el desarrollo de capacidades que facilitan la adaptación; en segundo lugar el principio de retención y difusión, según el cual durante el proceso evolutivo la empresa adquiere bien a nivel externo o a través de procesos de innovación, capacidades y competencias de sus trabajadores que tratará de transferirlos a la organización; en tercer lugar el principio de lucha por la supervivencia, el cual explica que dada la competencia con otras organiza-ciones por recursos escasos, la empresa aprovecha períodos de bonanza para adquirir recursos tangibles e intangibles para períodos de crisis.

Blumental y Haspeslagh (1994) consideran que el cambio impacta diversos aspectos de la organización: una perspectiva de mejoramiento de operaciones afectará los costos, la calidad y el servicio; una mirada estratégica impactará los objetivos del negocio, la obtención de ventaja competitiva sostenida, la realineación de las aptitudes de los integrantes de la empresa y el desarrollo y aprovechamiento de las capacidades organizacionales; un enfoque de autorrenovación corporativa afectará la forma como la organización percibe los negocios.

Para operacionalizar esta variable en la investigación se indagó la presencia de diez (10) ítems correspondientes a cinco variables: innovaciones (productos, procesos o servicios), cambios en la tecnología, cambios culturales (modificaciones significativas en 
valores, expectativas, políticas), reestructuraciones (fusiones, adquisiciones, reducción o crecimiento, cambios legales, reorganizaciones o modificaciones importantes en la alta dirección) y el reconocimiento de nuevos e importantes competidores.

Los anteriores aspectos teóricos permiten formular la hipótesis de que los cambios cuando se convierten en transformaciones organizacionales generan nuevas capacidades que fortalecen la ventaja competitiva de las empresas. Ahora bien, en el siguiente apartado analizaremos sobre qué capacidades se espera que impacte dicho cambio.

\subsection{Capacidades críticas como ventajas competitivas}

Para comprender el concepto de capacidades se recurre a la teoría de la empresa basada en recursos (Wernerfelt, 1984, p. 171-180), la cual trasciende la explicación tradicional de la competitividad como el aprovechamiento de imperfecciones del mercado y trata de comprenderla como el equilibrio entre las oportunidades que el entorno ofrece, mediadas por un conjunto de recursos y capacidades internas que la organización desarrolla y aprovecha. Una consecuencia inmediata de esta teoría emergente es que se valora tanto la oferta de un buen portafolio (productos y servicios con calidad y a precios competitivos), como la disponibilidad y utilización que haga de los recursos para generar nuevos productos y mercados (Penrose, 1959).

En este contexto, las capacidades se refieren a la competencia de una empresa para desplegar los recursos, combinándolos apropiadamente y usando procesos organizativos para producir un fin deseado (Amit y Schoemaker, 1993) que se refleje en un desempeño superior con respecto a las otras organizaciones (Lado y Wilson, 1994). Las capacidades son básicamente consecuencia de la acción gerencial para movilizar recursos mediante la generación de rutinas organizativas y una cultura abierta al cambio, resultado de un proceso de aprendizaje colectivo (Cuervo, 1995).

Dado que no todos los recursos ni las capacidades pueden considerarse críticas, en la presente investigación se hace referencia a éstas como las capacidades centrales que tienen posibilidad de hacer la diferencia competitiva en un entorno turbulento, es decir, que contribuyen a la construcción de ventaja competitiva sostenida y aumentan el desempeño organizacional. Leonard-Barton (1992, p. 111-115) reconoce cuatro dimensiones en las capacidades centrales de una organización (1) conocimiento y habilidades de los empleados, (2) sistemas técnicos, que contribuyen a acumular, codificar y estructurar conocimiento tácito, (3) sistemas de dirección que incluyen cambios formales e informarles para crear y controlar el conocimiento y (4) normas y valores asociados con el proceso de creación y control del conocimiento.

\subsection{Cambio y capacidades críticas (competitivas)}

Morgan (1997) ha definido el desarrollo de capacidades críticas como el proceso mediante el cual las personas, grupos y organizaciones mejoran sus habilidades para llevar a cabo sus funciones y alcanzar los resultados deseados a través del tiempo; esto implica que las capacidades críticas se crean gradualmente y se refuerzan durante largos períodos de tiempo (Ghetwat, 2000) y que están relacionadas con el entorno, pues la generación de capacidades es provocada por el ambiente en el cual se insertan las 
organizaciones y sus miembros (Motta, 2001); pero a su vez ambientes turbulentos demandan capacidades de anticipación y adaptación (Jiménez, 2002, p. 7-20), en otras palabras la capacidad crítica está llamada a lograr el ajuste estratégico con el ambiente cambiante (Teece, 1997).

La revisión de la literatura especializada arroja al menos seis capacidades críticas que teóricamente pueden ser desarrolladas en los procesos de cambio: flexibilidad, gestión del capital humano, liderazgo, aprendizaje organizacional, relaciones con el cliente y claridad estratégica.

La flexibilidad se refiere a la adaptabilidad sistémica de una empresa a cambios en su entorno competitivo, como ciclos económicos, innovaciones tecnológicas, modificaciones en la estructura y dinámica de los mercados, cambios en los gustos y preferencias del consumidor, entre otros. Esta capacidad se manifiesta de tres modos: flexibilidad en la gestión estratégica, financiera y comercial; flexibilidad en la gestión productiva y flexibilidad laboral.

La flexibilidad es una capacidad que ayuda a construir ventaja competitiva dado que al reducir ciclos y aumentar la oportunidad aumenta valor para el cliente, coadyuva a la reducción de costos y vuelve la organización más fluida y dinámica (Chowdhurry, et.al., 2000; Mendoza, 2000). Esta variable fue operacionalizada a través de los ítems: solución oportuna a las necesidades del cliente, respuesta rápida a cambios del mercado e implementación de procesos de mejora continua.

Uno de los campos organizacionales que mayor influencia está adquiriendo en el nuevo contexto empresarial es la gestión del talento humano, por su capacidad para generar ventaja competitiva y mejorar el desempeño organizacional (Pfeffer, 1996; Ulrich, 1997). Al respecto Delaney y Huselid (1996, p. 39, 949-969) constataron que las prácticas que afectan las habilidades de los empleados, su motivación y la estructura del trabajo están relacionadas positivamente con el desempeño de las organizaciones.

Wright, McMahan y Williams (1994, p. 301326) encontraron que los recursos humanos (grupo total de capital humano bajo el control de una empresa) tienen alta probabilidad de ser fuente de ventaja competitiva sostenida por cuanto tienen mayor probabilidad que otros recursos de ser inimitables, insustituibles, valiosos y escasos.

La capacidad de gestión del talento humano en la presente investigación tiene relación con la posibilidad de aumentar el capital humano de la organización pero sobre todo de aprovechar el potencial que puede generar el compromiso de las personas frente su organización si éstas consideran que su éxito personal está ligado al de la compañía y a su capacidad de actuar (estar empoderado). Esta variable se definió operacionalmente de la siguiente manera: existencia de espacios para aplicar nuevas ideas, posibilidad de desarrollo de las personas al interior de la empresa y empoderamiento de los empleados.

Otro factor importante es el liderazgo, entendido como una capacidad gerencial para adquirir, desarrollar y desplegar los recursos organizacionales, así como para convertir esos recursos en productos y servicios valiosos y entregar valor a los grupos de interés de la organización (Lado y Wilson, 1994).

El liderazgo se refleja en cuestiones tangibles como el permanente surgimiento de ideas, proyectos e innovaciones; apertura mental y clima propicio para recibir y aceptar esas 
nuevas ideas y en la constitución de un ambiente adecuado para que surjan personas capaces de «jalonar» la empresa, algunas de sus áreas o proyectos específicos.

Una capacidad que desde los trabajos de Senge (1992) fue aceptada como fundamental para el desarrollo empresarial es el aprendizaje organizacional. La teoría reconoce dos tipos de aprendizaje, el denominado de bucle sencillo relacionado con los conocimientos y habilidades que adquieren las personas de una empresa para cumplir su trabajo y sus responsabilidades. Este se fundamenta en procesos de formación y entrenamiento. En segundo lugar, aún más importante, el aprendizaje de bucle doble, que además de lo anterior forma a las personas para cuestionar lo pre-existente, reflexionar y generar nuevos significados sobre sus acciones.

Este último tipo de aprendizaje puede «reforzar la flexibilización organizacional al permitirle a los miembros pensar y responder de forma divergente a los cambios en el ambiente interno y externo" (Lado y Wilson, 1994). Por ello esta variable se define operacionalmente como: la existencia de programas de capacitación, el manejo de la información para mejorar conocimientos organizacionales y la posibilidad de que los nuevos empleados adquieran y desarrollen conocimientos en la acción-reflexión de su trabajo.

Las relaciones con el cliente son una capacidad cada vez más exigida y más compleja en la gestión de las organizaciones. La fidelización no se genera simplemente por hábitos de consumo, o sea, por una relación centrada en el producto: se requiere una relación más amplia, sensible y estratégica. Esto implica un esfuerzo significativo para obtener información sobre el cliente y poderle responder de manera personalizada. Pero esto involucra una importante inversión tecnológica para mejorar la comunicación y la interacción.

La claridad estratégica hace referencia a la capacidad directiva para formular una estrategia competitiva, articularla con la estructura y la cultura de la organización, comunicarla a todos los miembros de la empresa y crear las condiciones para que se pueda ejecutar (Lado y Wilson, 1994). La perspectiva estratégica supone que las compañías crecen y alcanzan nuevos resultados e impactos en el ambiente como fruto de la capacidad de adaptación constante (Motta, 2001). Como lo plantean Hamel y Prahalad (1995) se necesita contar con una postura estratégica basada en la visión, la misión y los objetivos y definir qué cambios se requieren introducir en la plataforma competitiva, para alcanzar una ventaja que garantice desempeños superiores.

En síntesis, la claridad estratégica es concebida en esta investigación en relación con la construcción y las prácticas de un marco orientador con principios, conceptos, enfoques y modelos que incidan en la forma de pensar y actuar de la organización. El concepto se planteado tomando tres factores: que existan y se conozcan planes de desarrollo futuro de la empresa, que estén claramente establecidos los objetivos y metas y que las personas sientan que su proyecto de vida está ligado al de la empresa.

A partir de lo encontrado en la revisión teórica puede pensarse en una relación del cambio con la posibilidad de generar y fortalecer las capacidades mencionadas por parte de las organizaciones. En el siguiente numeral se constatará lo hallado en las empresas medianas y grandes del sector de las confecciones en el Eje Cafetero. 


\section{CAMBIO Y GENERACIÓN DE CAPACIDADES COMPETITIVAS EN EL SECTOR DE CONFECCIONES DEL EJE CAFETERO. HALLAZGOS Y SU INTERPRETACIÓN}

Este análisis de resultados se presenta desde dos perspectivas: en primer lugar interesa conocer cómo se comportan las variables en la población objeto de estudio; es decir, se hace un análisis descriptivo del ambiente de cambio que han vivido las empresas y el estado de las variables dependientes: flexibilidad, gestión del capital humano, liderazgo, aprendizaje organizacional, relaciones con el cliente y claridad estratégica. En segundo lugar se analizan los resultados del análisis de varianza para establecer la validez de la hipótesis, es decir, qué tanto ha incidido el ambiente de cambio sobre las variables dependientes mencionadas.

\subsection{Descripción de las varia- bles en el sector de las confecciones}

El ambiente de cambio se percibe en un nivel medio alto por parte de los encuestados en las empresas de confecciones del Eje Cafetero, de manera que en una escala de 1 a 5 calificaron en promedio con 4.2 la presencia de cambio en sus organizaciones.

El ambiente de cambio fue valorado de manera muy homogénea entre las diferentes empresas, distribuyéndose en un rango entre 3.9 la que menos percibe una presión de cambio y 4.5 aquella que más siente el cambio como factor importante en su organización. En los niveles superiores se ubicaron empresas medianas con clientes extranjeros.

Como puede observarse en la Tabla 1 los factores donde los directivos empresariales perciben un mayor efecto del cambio está en la aparición de nuevos e importantes competidores y en las innovaciones de productos procesos y servicios, siendo menos notorios los cambios tecnológicos y culturales de la organización.

Tabla 1. Valoración de los factores de cambio en las organizaciones

\begin{tabular}{|l|c|}
\hline \multicolumn{1}{|c|}{ FACTORES DE CAMBIO ORGANIZACIONAL } & VALORACIÓN \\
\hline Innovaciones (procesos, productos o servicios) & 4.4 \\
\hline Tecnología & 4.1 \\
\hline Culturales (valores, expectativas, políticas) & 4.1 \\
\hline $\begin{array}{l}\text { Reestructuraciones (fusiones, adquisiciones, reducción } \\
\text { o crecimiento, cambios legales, reorganizaciones internas } \\
\text { o cambios en alta dirección) }\end{array}$ & 4.2 \\
\hline Nuevos competidores & 4.5 \\
\hline Percepción general de cambio (promedio) & 4.2 \\
\hline
\end{tabular}


Figura 1. Desarrollo de las capacidades competitivas en las empresas de confecciones

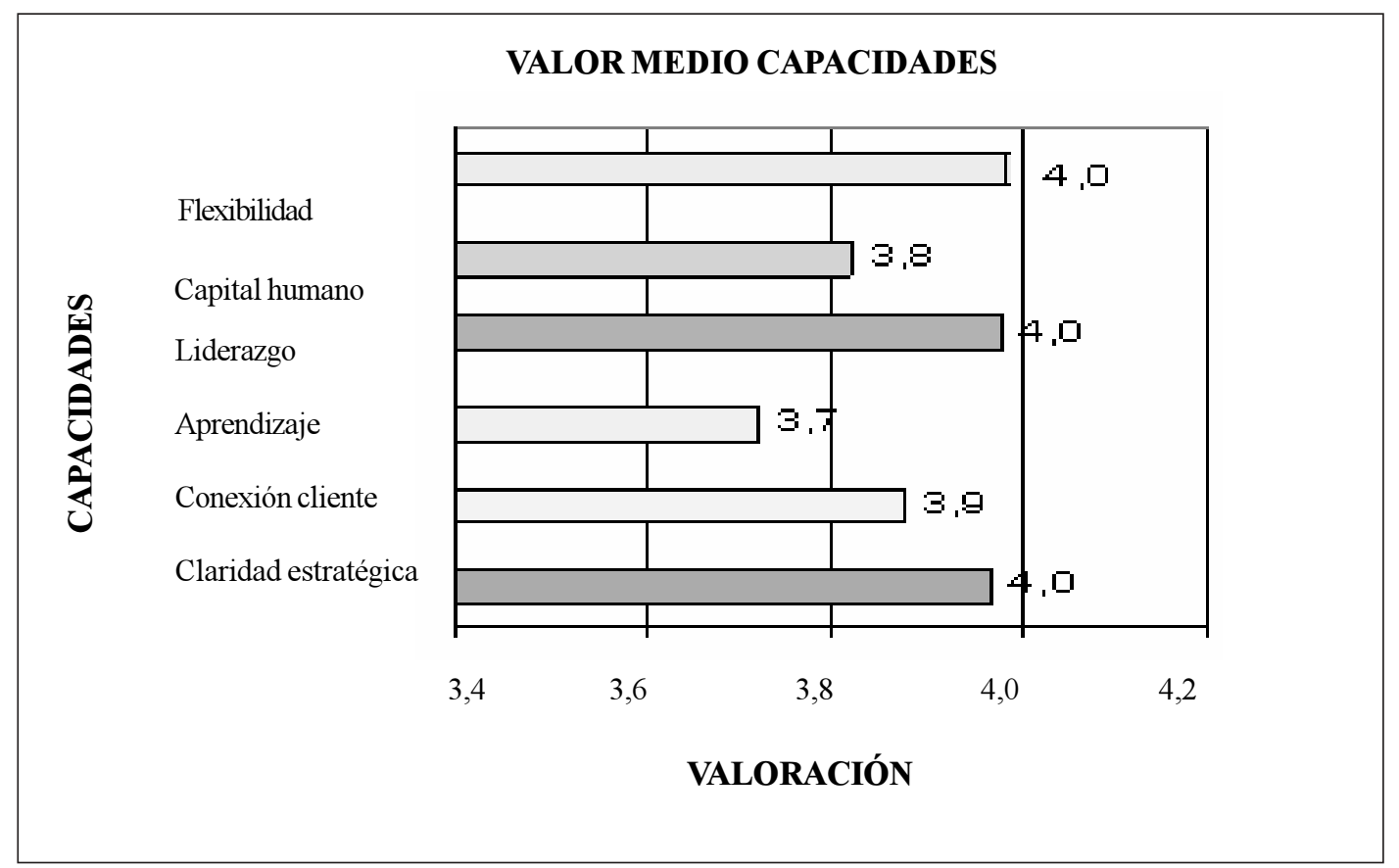

Fuente: Resultados de la investigación

En cuanto al desarrollo de las capacidades críticas, en la Figura 1 se observa la valoración promedia de los directivos del sector de las confecciones. Sobresale la claridad estratégica, la flexibilidad y el liderazgo como las más desarrolladas en las empresas estudiadas; las menos desarrolladas son el aprendizaje organizacional y la gestión del capital humano.

La flexibilidad se manifiesta en la agilidad para responder a las necesidades de los clientes y a las exigencias del mercado $y$-en menor medida- en la habilidad para diseñar y ejecutar mejoras de procesos. En cuanto a la capacidad de incrementar el capital humano, poco desarrollada en las empresas analizadas, se percibe una falencia en el empoderamiento de las personas que se compensa con un ambiente propicio para proponer e implementar nuevas ideas, aunque no existen las mejores condiciones para el progreso de las personas.
De otro lado, la capacidad de liderazgo está parcialmente desarrollada pues aunque se acepta que existen personas visionarias no siempre existe la capacidad para impulsar y liderar nuevos proyectos.

El aprendizaje es la capacidad menos desarrollada en las empresas objeto de estudio, por la incidencia de las limitaciones en la formación y la gestión de la información.

En contra de lo esperado la capacidad de relacionarse con el cliente tampoco es una de las capacidades más altas y aunque se reconoce que se ha invertido en tecnología de comunicaciones para mejorar esta relación, los directivos encuestados aún perciben que no se tiene la suficiente oportunidad en atender sus demandas. Por último la claridad estratégica muestra que se ha avanzado en la divulgación de los objetivos y en tratar de 
lograr el compromiso de todos los actores de la empresa con ellos, pero al parecer existen dificultades para lograr una visión compartida de futuro en las empresas.

\subsection{Prueba de hipótesis}

Teniendo en cuenta que el valor crítico para F con un nivel de significación del 0.05 es de 3.982 y considerando los resultados arrojados por el ANOVA (Tabla 2) resulta imposible rechazar la hipótesis nula; es decir, se carece de evidencia empírica para rechazar una posible relación de asociación entre el cambio y cada una de las seis capacidades críticas estudiadas.

Significa lo anterior que las empresas que han sido sometidas a presiones de cambio han

Tabla 2. Análisis de varianza de las capacidades críticas en relación con el cambio

\begin{tabular}{|l|c|l|l|l|}
\hline CAPACIDAD & V/R PROMEDIO & VARIANZA & F & PROBAB. \\
\hline Flexibilidad & 3.971 & 0.257 & 5.600 & 0.0207 \\
\hline Capital Humano & 3.819 & 0.329 & 12.111 & 0.0009 \\
\hline Liderazgo & 3.981 & 0.248 & 5.317 & 0.0242 \\
\hline Aprendizaje & & & 19.136 & 0.00004 \\
\hline Relaciones con el cliente & & & 10.091 & 0.0022 \\
\hline Claridad estratégica & & & 6.602 & 0.0124 \\
\hline
\end{tabular}

Fuente: Resultados de la investigación

tenido la oportunidad de desarrollar algunas de las capacidades críticas para ser competitivas (liderazgo, flexibilidad y claridad estratégica), lo que les facilitará afrontar nuevas situaciones de cambio, así como incrementar sus recursos y capacidades, que según lo formulado por Ventura (1996) se constituyen en una característica de la estrategia dinámica de la empresa. Coincide esto con lo afirmado por la teoría de la empresa basada en recursos, que «se apoya en el supuesto de que el fortalecimiento de la empresa no está dado tanto en el crecimiento de sus productos actuales sino en el potencial de crear nuevos productos y penetrar nuevos mercados, es decir, por la disponibilidad y utilización que haga de sus recursos" (Calderón, 2006) y por la habilidad que tenga durante este proceso de desarrollar nuevas capacidades que a su vez se constituyen en sustento para fortalecer su estrategia competitiva y agregar valor a la organización.

Por último, preocupa que las relaciones más débiles se presentan en dos capacidades que están estrechamente relacionadas con la gestión humana en las organizaciones: el aprendizaje y el desarrollo del capital humano, lo cual refleja una visión tradicional del manejo de personal, que pierde la potencialidad, comprobada en muchas investigaciones, del talento humano para la generación de ventaja competitiva sostenida (Mc Williams, et.al., 2001, 1-24; Barney y Wrigth, 1998 ) y para la generación de valor (Becker, et.al., 1998; Buyens y de Vos, 1999). 
Tabla 3. Pruebas de autocorrelación

\begin{tabular}{|c|c|c|c|}
\hline Variable & Prueba Tolerance & $\begin{array}{l}\text { Prueba de } \\
\text { significación } \\
\text { del cambio en f }\end{array}$ & Prueba $R_{2}$ \\
\hline $\begin{array}{l}\text { Valores } \\
\text { esperados }\end{array}$ & $\begin{array}{l}\text { Valores alejados } \\
\text { de cero }\end{array}$ & $\begin{array}{l}\text { Significación } \\
\text { no mayor } 0.10\end{array}$ & $R_{2}$ mayor a 0.1 \\
\hline \multirow[t]{2}{*}{ Flexibilidad } & $\begin{array}{l}\text { Error típico de } \\
\text { estimación }=0.42\end{array}$ & $S=0.01$ & $R_{2}=0.2949$ \\
\hline & ok. & ok & ok \\
\hline \multirow[t]{2}{*}{ Capital humano } & $\begin{array}{l}\text { Error típico de } \\
\text { estimación }=0.578\end{array}$ & $S=0.355$ & $R_{2}=0.0249$ \\
\hline & ok. & autocorrelación & autocorrelación \\
\hline \multirow[t]{2}{*}{ Aprendizaje } & $\begin{array}{l}\text { Error típico de } \\
\text { estimación }=0.568\end{array}$ & $S=0.784$ & $R_{2}=0.035$ \\
\hline & ok. & autocorrelación & autocorrelación \\
\hline \multirow[t]{2}{*}{ Liderazgo } & $\begin{array}{l}\text { Error típico de } \\
\text { estimación }=0.4395\end{array}$ & $S=0.002$ & $R_{2}=0.2671$ \\
\hline & ok. & ok & ok \\
\hline \multirow[t]{2}{*}{$\begin{array}{l}\text { Conexión } \\
\text { cliente }\end{array}$} & $\begin{array}{l}\text { Error típico de } \\
\text { estimación }=0.5327\end{array}$ & $S=0.486$ & $R_{2}=0.0155$ \\
\hline & ok. & autocorrelación & autocorrelación \\
\hline \multirow[t]{2}{*}{$\begin{array}{l}\text { Claridad } \\
\text { estratégica }\end{array}$} & $\begin{array}{l}\text { Error típico de } \\
\text { estimación = } 0.472\end{array}$ & $S=0.439$ & $\mathrm{R}_{2}=0.0191$ \\
\hline & ok. & autocorrelación & autocorrelación \\
\hline
\end{tabular}

Fuente: Resultados de la investigación.

De otra parte, con el fin de analizar la relación de causalidad entre el cambio y las capacidades críticas se calculó la ecuación de regresión, para lo cual se realizó inicialmente la prueba de Durbin-Watson con el fin de evitar las denominadas autocorrelaciones (en la Tabla 3 se muestran sus resultados), dando como consecuencia que las capacidades de capital humano, aprendizaje, relación con el cliente y claridad estratégica están autocorrelacionadas, por lo tanto el análisis de regresión fue hecho con las otras dos capacidades.

Como se observa en la Tabla 4 se puede afirmar que el cambio, con un nivel de significación del 0.05 , se acepta como predictor de la capacidad de flexibilidad (explica al menos el $28.6 \%$ ) y la capacidad de liderazgo (explica un $26.7 \%$ ). Sin embargo esto solo debe tomarse como una aproximación exploratoria pues sin duda habría que considerar otras variables, además del cambio, que pueden incidir sobre la generación de capacidades organizativas. Pero el indicio hallado en este trabajo puede alertar a los gerentes para aprovechar los procesos de transformación organizacional como insumo importante para fomentar y desarrollar nuevas capacidades (al menos, específicamente, liderazgo y flexibilidad) y a los investigadores para indagar en nuevos trabajos sobre estas relaciones. 
Tabla 4 Prueba de validez para la ecuación de regresión

Flexibilidad $=0.709 \times$ Cambio +0.963 , con un $R_{2}$ de 0.286 y un valor $t=3.370$

Profundidad de liderazgo $=0.6524 \times$ Cambio +1.222 , con un $R_{2}$ de 0.267 y un valor $t=3.370$

Valor de significación: 0.05

Fuente: Resultados de la investigación

El cambio es una cadena de ciclos que genera inesperadamente oportunidades para comenzar transformaciones internas, pero es la organización la que decide si las aprovecha o no; de ella es la decisión de cambiar soltando algunas anclas y abriendo la posibilidad incierta de que el eslabón siguiente represente crecimiento y fertilidad. Pero sin duda este contexto ha estimulado la exigencia de mayor valor para el cliente, la reducción de los costos unitarios y la necesidad de competitividad.

Las capacidades, por otra parte, se refieren a la competencia de una empresa para desplegar sus recursos, usualmente en combinación, usando procesos organizativos para producir un fin deseado e incorporando interacciones complejas entre las personas.

En principio, la revisión teórica permitió plantear una posible asociación entre el cambio y algunas capacidades organizativas, explícitamente flexibilidad, gestión del capital humano, liderazgo, aprendizaje organizacional, relaciones con el cliente y claridad estratégica.

Posteriormente, la contrastación empírica arrojó al menos cuatro conclusiones: (1) todas las empresas medianas y grandes de confecciones del Eje Cafetero que fueron analizadas perciben una fuerte presión de cambio generada por factores externos que les ha obligado a realizar transformaciones internas; (2) los encuestados coinciden en reconocer la presencia de todas las capacidades organizativas estudiadas, aunque dos de ellas con un significativo menor desarrollo (capital humano y aprendizaje organizacional); (3) la verificación de hipótesis arroja que existe evidencia empírica para afirmar que el cambio está asociado con el desarrollo de todas las capacidades organizativas estudiadas; y (4) puede afirmarse que existe una relación de causalidad cambio-capacidades, en el caso de flexibilidad y liderazgo.

Estos resultados reafirman la importancia, para los gerentes y directivos empresariales, de aprovechar el cambio no sólo para lograr actualizaciones tecnológicas, de procesos o de técnicas organizacionales, sino para desarrollar sus capacidades y sus recursos, que a su vez se convertirán en nuevos insumos para las siguientes formulaciones estratégicas. Así mismo se les presenta una oportunidad de reflexión frente al escaso desarrollo de capacidades asociadas a la gestión humana como son el aprendizaje organizacional y el capital humano.

De igual forma, para los investigadores se constituye en un paso que abre más perspectivas para estudios relacionados que permitan confirmar o refutar estos hallazgos, así como para profundizar en aspectos como las relaciones entre cambio y flexibilidad o entre cambio y liderazgo. 


\section{Bibliografía}

AMIT, R. \& SCHOEMAKER, P. J. H. (1993). Strategic assets and organizational rent. Strategic Management Journal.

BARNEY, J. (1991). Firms resources and sustained competitive advantage. Journal of Management, Vol 17.

BARNEY, J. B. \& WRIGTH, P. M. (1998). On Becoming a strategic partner: The role of Human resources in gaining competitive advantage. Human Resource Management, vol. 37.

BECKER, B. E.; HUSELID, M. A.; PICKUS, P. S.; y SPRATT, M. F. (1998). Los RH como fuente de valor para los accionistas: Investigación y recomendaciones. En: ULRICH, O.; LOSEY, M. R.; y LAKE G. EI Futuro de la Dirección de Recursos Humanos. Barcelona, Gestión 2000.

BLUMENTAL, B.; and HASPESLAGH, P. (1994). Toward a definition of corporate transformation. Sloan Management Review v (35) n3 ,Spring 1994.

BOXALL, P. F. (1996). The strategic human resource management debate and the resource-based view of the firm. Human Resource Management Journal, 6(3).

BUYENS, D.; and DE VOS, A. (1999). The added value of the $H R$ department. In: International HRM. Contemporary Issues in Europe. Londres, Ed. Brewster and Harris.

CALDERóN, G. (2006). Competencias distintivas en las PYMES: Un aporte desde gestión humana. Revista Innovar Vol. 15 No. 1(27). Enero - Junio, Bogotá, Universidad Nacional de Colombia (En prensa).

CHILD, J. \& KIESER, A. (1981). Develpment of organizations over time. Handbook of Organizational Design. Nystrom, New York, Oxford.

CHOWDHURRY, S.; SENGE, P.; PRAHALAD, C.; \& ULRICH, D. (2000). Managament siglo XXI. Santa fe de Bogotá: Prentice Hall.

CUERVO, A. (1995). La dirección estratégica de la empresa: Dirección de empresas de los noventa. Madrid: Editorial Civitas.

DELANEY, J. T.; \& HUSELID, M. A. (1996). The impact of human resource management practices on perceptions of organizational performance. Academy of Management Journal.

GHETWAT, P. (2000). La estrategia en el panorama de los negocios: Textos y casos. México, Prentice Hall.

GREWAL, R.; \& TANSUHAJ, P. (2001). Building organizational capabilities for managing economic crisis: The role of market orientation and strategic flexibility. Journal of Marketing, Apr2001, Vol. 65 Issue 2.

HAECKEL, S. (2002). La empresa adaptable: En la era de la información asimilar los cambios no es todo, es lo único. México, Mac Graw-Hill. 
HAMEL, G.; y PRAHALAD, C. (1995). Compitiendo por el futuro. Madrid, Editorial Ariel.

HANNA, M.; y FREEMAN, J. (1977). The population ecology of organizations. American Journal Sociology, No 82.

JIMÉNEZ, A. (2002). Modelo de análisis de competitividad de la empresa. Revista Economía y Administración No. 58.

KELVEY, M.; \& ALDRICH, H. (1983). Populations, natural selection and applied organizational science. Administrative Science Quarterly, No 28.

LADO, A. A.; \& WILSON, M. C. (1994). Human resource systems and sustained competitive advantage: A competency based perspective. Academy of Management Review, 19 (4).

LEONARD-BARTON, D. (1992). Core capabilities and core rigidities: A paradox in managing new product development. Strategic Management Journal, No 13.

MC WILLIAMS, A. D.; FLEET, V.; \& WRIGHT, P. (2001). Strategic management of human resources for global competitive advantage. Journal of Business Strategies,18(1).

MENDOZA, J. M. (2000). Los desafíos gerenciales de la globalización. Revista Pensamiento y Gestión, Uninorte; No. 8, Julio.

MORGAN, P. (1997). The design and use of capacity development indicators. Paper prepared for the political and social policies division, policy branch, CIDA. Hull; Canadian International Development Agency (CIDA).
MOTTA, P. R. (2001). Transformación organizacional. Bogotá, Alfaomega S.A.

NAMAKFOROOSH, M. (2002). Metodología de la investigación. México: Limusa.

PENROSE, E. T. (1959). The theory of the growth of the firm. New York: Wiley. Version en castellano: La teoría del crecimiento de la empresa, Editorial Aguilar, Madrid, 1962.

PFEFFER, J. (1996). Ventaja competitiva a través de la gente. Cómo desencadenar el poder de la fuerza de trabajo. México. Editorial Continental S.A.

SENGE, P. (1992). La quinta disciplina. Cómo impulsar el aprendizaje en la organización inteligente. Barcelona: Editorial Granica.

TEECE, D. J.; PISANO, G.; \& SHUEN, A. (1997): «Dynamic capabilities and strategic management». Strategic Management Journal, Vol 18:7.

ULRICH, D. (1997). Recursos Humanos Champions: Cómo pueden los recursos humanos cobrar valor y producir resultados. Buenos Aires: Ediciones Granica.

VALLES, M. S. (1999). Técnicas cualitativas de investigación social. Reflexión metodológica y práctica profesional. Madrid: Editorial Síntesis S.A.

VENTURA, J. (1996). Análisis dinámico de la estrategia empresarial: un ensayo interdisciplinario. Oviedo: Universidad de Oviedo.

VIVIANCO, F.; NAWAR, M. E.; y NOVOA, C. (2002). Vinculaciones empresariales en la industria de la confección. Documento del 
BID Colombia. Mayo. Disponible en http:// www.iadb.org/exr/ Consultado en Noviembre de 2005.

WERNERFELT, B. (1984). A resource - based view of the firm. Strategic Management Journal, 5.
WRIGHT, P. M.; Mcmahan, G.C.; \& MCWILLIAMS, A. (1994). Human resources and sustained competitive advantage: $A$ resourced-based perspective. International Journal of Human Resource Management, 5 (2). 
